Research Article

\title{
Application of Configuration Force to Dynamic Crack of Shale under Hydration
}

\author{
Heng Fan $\mathbb{D}^{1},{ }^{1}$ Xiaojiao Zhao, ${ }^{1}$ Yu Ji, ${ }^{1}$ Zhifeng Zhao, ${ }^{1}$ and Zhan $Q u^{2}$ \\ ${ }^{1}$ Safety Engineering, Xi'an Shiyou University, Dianzi No. 2 Road, Xi'an, China \\ ${ }^{2}$ Petroleum Engineering, Xi'an Shiyou University, Dianzi No. 2 Road, Xi'an, China \\ Correspondence should be addressed to Heng Fan; fan_h@xsyu.edu.cn
}

Received 2 March 2021; Revised 14 July 2021; Accepted 28 August 2021; Published 18 September 2021

Academic Editor: Jianguo Wang

Copyright (c) 2021 Heng Fan et al. This is an open access article distributed under the Creative Commons Attribution License, which permits unrestricted use, distribution, and reproduction in any medium, provided the original work is properly cited.

The natural microdefects of shale and the expansion of microcracks under hydration and overlying rock loadings are important for the wellbore stability. According to the conservation of energy, the force of the microdefects and microcracks under finite deformation is studied by the method of configuration force through the migrating control volume in the spatial observer. Under the hydration stress and rock pressure, the equation of hydration stress and its work in reference configuration has been obtained, and the equations of configuration forces and configuration moment have been established as a consequence of invariance under changes. The relationship between the configuration and deformation forces is determined by the second law. The energy dissipation equation of the crack tip has been deduced, which shows that the projection of the concentrated internal configuration body force at the crack tip in the opposite direction of the crack is equal to the energy dissipation of the crack tip per unit length. The inertial and internal parts of the concentrated configuration body force at the crack tip have been derived; it is indicated that the internal configuration force plays a leading role in the irreversible fracture process. Moreover, the energy release rate of shale under hydration is proved to depend on constitutive responses and hydration stress. In the theoretical system of configuration force, the migrating control volume at the crack tip contains inclusions, microcracks, microvoids, and heterogeneity of the rock itself. We use the configuration force theory to solve the problem of rock crack propagation and rock fracture. The factors considered are more comprehensive, which can better reflect the actual situation and provide a theoretical basis for the study of wellbore stability.

\section{Introduction}

The instability of the shaft lining is closely related to the properties of the rock itself. Shale contains various defects, inclusions, microcracks, and microvoids. Under the action of external force and hydration, when the surrounding rock of the shaft wall cannot bear the energy accumulated in the rock, microcracks or original microcracks start to crack, extend, communicate with each other, and then form macrocracks. This process can be seen as energy dissipation and release. Wellbore stability is mainly studied from the three aspects of mechanics, chemistry, and multiphysical coupling. Mechanical research studies the stress distribution around the wellbore and the influence of anisotropy on wellbore stability from the perspective of mechanical energy [1-4]. Chemical research studies the influence of hydration of drilling fluid filtration and shale on the rock strength from the perspective of chemical energy, which leads to borehole wall falling or collapse [5-8]. Mechanical energy, chemical energy, formation stress, and other factors are comprehensively considered in the multiphysical coupling study of shale $[9,10]$. Finally, it is concluded that the chemical energy of shale hydration caused by the contact between shale and drilling fluid is the main reason for wellbore instability.

In the process of drilling, the pressure of overlying rock produces mechanical energy on the surrounding rock of the shaft wall, and the infiltration and hydration reaction of drilling fluid filtration in the formation produce chemical energy on the surrounding rock of the shaft wall. When the accumulation of this energy exceeds the bearing limit of rock, a fracture will occur, resulting in wellbore instability. Santos [11] puts forward the energy model of wellbore 
stability according to the law of energy conservation. According to the law of conservation of energy, Griffith [12] integrates the strain energy density around the rock crack and obtains the crack growth when the elastic potential energy release rate is greater than the surface energy increase rate. On this basis, Irwin [13] considered the influence of material plasticity on crack growth. Based on the principle of strain energy equivalence, Kemeny [14] studied the failure process of rock containing mode II crack and established a constitutive model. Basista [15] studied the propagation process of slip mode crack from the angle of energy transformation. Xie et al. [16] discussed the relationship between energy release and dissipation, rock strength, and overall failure from the perspective of mechanics and thermodynamics. Chen et al. [17] analyzed the transformation of energy and gave the calculation formula of surface energy, elastic potential energy, and kinetic energy of rock fracture. Zhang [18], Zhang [19], and Zhou [20] studied the relationship between energy dissipation and rock failure from different perspectives. Zhao [21] applied entropy theory and energy principle to the study of hydraulic fracture initiation and extension and established the entropy change equation and fracture initiation model of hydraulic fracture. Zhao [22] used an analyzed numerical simulation method to analyze the characteristics of energy accumulation and release at the tip of shale hydraulic fracture. Li [23] carried out a study on the energy transformation law in the process of hydraulic fracturing and pointed out that the rock mass damage and fracture propagation are the results of the transformation of external load work and fracturing gravity potential energy. According to the conservation of energy, it is an effective method to solve the problem of rock failure.

However, according to the law of conservation of energy, there are few reports on the application of configuration force theory to the analysis of rock crack propagation. The concept of configuration force could be traced back to Eshelby [24-27], who put forward the energy-momentum tensor. On this basis, configuration force has been developed to study the evolution and defects of material structures [28-32]. Then, the velocity of migrating control volume was introduced to configuration force by Gurtin [30-32], so the configuration force could be applied to the dynamic crack. However, the equilibrium equation of configuration force was established under pure mechanical loading. It was confirmed that the configuration force at the crack tip was the driving force of crack propagation [30-32]. In the case of thermoelastic fracture, it was deduced that the crack driving force was consistent with the well-known energy release rate [33-36]. Lately, configuration force was used to study the interplay between the crack driving force and the fracture evolution [37]. Eshelby stress has been applied to analyze the configuration force opposing the crack tip motion using the tensor and local force balance law in cracked and heterogeneous domains [38]. The configuration force could be also viewed as the resultant of the contact forces acting on the perturbed shape of an object of substance equivalent to the defect and evaluated in the limit of the shape being restored to the primitive configuration [39]. The configuration force could be computed efficiently and robustly when a constitutive continuum model of gradient-enhanced viscoplasticity was adopted [40]. The small strain multiphasefield model accounting for configuration forces and mechanical jump conditions was constructed [41]. The theory of configuration force based on r-adaptive mesh refinement was discussed in the context of isogeometric analysis [42].

However, the systematic configuration force theory of shale under hydration following Gurtin's point of view is still absent. Therefore, this paper will follow the key point of Gurtin, as Gurtin considered the velocity of the defect evolution. In the present paper, the basic concept and laws of configuration force of shale under hydration are constructed, which will be applied to dynamic fracture of shale. The working of all loadings will be constructed, and the energy release rate of shale under hydration will be evaluated.

\section{Basic Theory}

2.1. Definition of Migrating Control Volume. In Gurtin's theory [30-32], the key point is migrating control volume. $B_{r}$ is a body where material points $X$ exist in the reference configuration (Figure 1); that is, $\mathbf{X} \in B_{r}$ and $t \in \mathbb{R} . y=$ $\bar{y}(\mathbf{X}, t)$ represents the position of the material points in the current configuration, $F$ is the deformation gradient, and $\dot{y}$ is the material velocity; consequently, $B_{t}=\bar{y}\left(B_{r}, t\right)$ denotes the whole body's deformation $B_{r}$ in the current configuration.

$R(t)$ represents the reference configuration of deformable-body (a subset of $B_{r}$ ), which takes up space for $R_{0}$ at the reference moment $t_{0}$. The control volume boundary $\partial R(t)$ in the reference configuration is in constant motion. Material points corresponding to the different times can be expressed as

$$
\mathbf{X}=\hat{X}\left(\xi_{1}, \xi_{2}, t\right) .
$$

The boundary velocity of the evolutionary control volume $\partial R(t)$ from the angle of the spatial observer is

$$
\mathbf{u}=\frac{\partial \hat{X}\left(\xi_{1}, \xi_{2}, t\right)}{\partial t}, \partial \hat{X}\left(\xi_{1}, \xi_{2}, t\right) \in \partial R(t),
$$

where $\mathbf{u}$ describes the evolutionary rate of defects, so this paper tends to use Gurtin's theory to study the crack propagation caused by hydration of shale. $\left(\xi_{1}, \xi_{2}\right)$ is the parameter to control the tangential evolution velocity of interface $\partial R(t)$, which can be freely chosen in the case that the normal component $U_{\partial R(t)}$ of the interface evolution velocity is unchanged.

$$
U_{\partial R(t)}=\mathbf{u} \cdot \mathbf{n},
$$

where $n$ is the unit normal vector of the boundary of the migrating control volume. $y=\bar{y}(X, t)$ represents the position of the material points in the current configuration. In this way, the points' position $X=\widehat{X}\left(\xi_{1}, \xi_{2}, t\right)$ of the control volume boundary $\partial R(t)$ in the current configuration is $y=\bar{y}\left(\widehat{X}\left(\xi_{1}, \xi_{2}, t\right), t\right)$. On this basis, we can define the interface deformation velocity caused by the migrating control volume. 


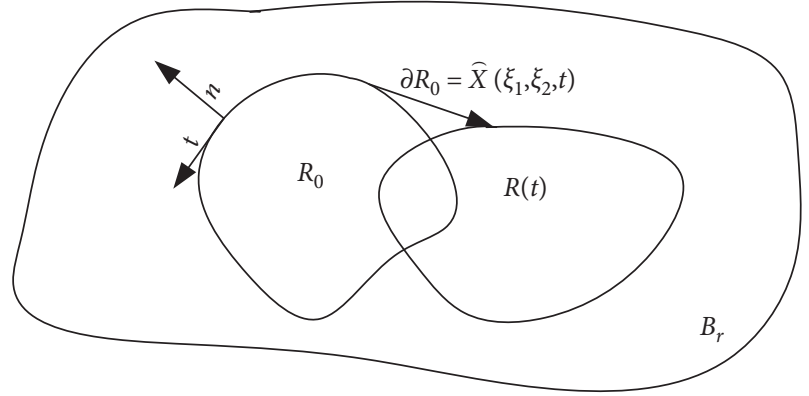

FIgURE 1: Diagrammatic sketch of the migrating control volume.

$$
\overline{\mathbf{u}}(\mathbf{X}, t)=\frac{\partial \widehat{y}\left(\widehat{\mathbf{X}}\left(\xi_{1}, \xi_{2}, t\right), t\right)}{\partial t}=\dot{y}+\mathbf{F u}
$$

where $\dot{y}$ is the motion rate of the material points. From the above model, it can be found that the velocity of interfacial deformation is the amount of motion that describes the motion of material points and the interaction between continuum deformation and interface evolution.

\subsection{Geometry and Motion Description of Cracked Bodies.} As shown in Figure 2, $B_{r}$ represents a closed region whose boundary is $\partial B_{r}, \ell(t)$ is a crack in $B_{r}$. Assuming that one of the end points $Z_{0}$ is fixed, the crack tip $\mathbf{Z}(t)$ is extended. The position of the reference time in the reference configuration is represented by $Z\left(t_{0}\right)$. The unit vector e represents the direction of crack propagation, and $\mathbf{m}(\mathbf{X})$ is the normal direction of the crack surface. $\mathbf{n}(\mathbf{X})$ is the normal direction of the migrating control volume containing crack tip $R(t)$.

Here, we define a special disk migrating control volume $D_{\delta}(t)$ with a crack tip (shown in Figure 2).

$$
D_{\delta}(t)=\left\{\mathbf{X} \in B_{r}:|\mathbf{X}-\mathbf{Z}(t)| \leq \delta\right\},
$$

where $\delta$ represents the radius of the disk. In the meantime, the following notations are given: $\ell_{\delta}(t)=\ell_{D_{\delta}}(t)=\ell(t)$ $\cap D_{\delta}(t), \ell_{R_{\delta}}(t)=\ell_{R}(t) \backslash \ell_{\delta}(t), \ell_{R}(t)=\ell(t) \cap R(t), R_{\delta}(t)=R$ $(t) \backslash D_{\delta}(t), \partial R_{\delta}(t)=\partial R(t) \cup \partial D_{\delta}(t)$.

The crack propagation velocity describes the position change of the crack tip in the reference configuration. It can be defined as

$$
\mathbf{v}(t)=\frac{\mathrm{d} \mathbf{Z}(t)}{\mathrm{d} t} .
$$

In fracture mechanics, the arbitrary field variable in the fixed frame $\Phi(\mathbf{X}, t)$ can be expressed as $\widehat{\Phi}(\mathbf{Y}, t)$ in the frame with the movement of the crack tip. It can be expressed as

$$
\widehat{\Phi}(\mathbf{Y}, t)=\Phi(\mathbf{X}, t) \text {. }
$$

The formula $Y=X-Z(t)$ expresses the position of material points $X$ with respect to the crack tip $Z(t)$. Then, the time derivative $\Phi^{\circ}(X, t)$ of the $\Phi(X, t)$ relative to the crack tip motion reference system can be defined as

$$
\Phi^{\circ}(\mathbf{X}, t)=\left.\frac{\partial \widehat{\Phi}(\mathbf{Y}, t)}{\partial t}\right|_{Y=\text { const }}=\dot{\Phi}(\mathbf{X}, t)+\nabla \Phi(\mathbf{X}, t) \cdot v .
$$

Taking $\Phi(\mathbf{X}, t)$ as $\mathbf{y}=\hat{y}(\mathbf{X}, t)$, the velocity of the reference point with $Y$ distance from the crack tip in the motion observation can be defined as

$$
y^{\circ}(\mathbf{X}, t)=\dot{y}(\mathbf{X}, t)+\mathbf{F v} .
$$

When $\mathbf{X} \longrightarrow \mathbf{Z}(t)$, (9) represents the velocity of the crack tip in the current configuration $\bar{v}(t)$, i.e., the deformation velocity at the crack tip.

$$
\overline{\mathbf{v}}(t)=\mathbf{y}^{\circ}(\mathbf{Z}(t), t)=\dot{y}(\mathbf{Z}(t), t)+\mathbf{F v} .
$$

The deformation velocity of crack tip $\overline{\mathbf{v}}(t)$ and the deformation velocity of migrating control volume interface $\overline{\mathbf{u}}$ are similar. Both of them are not the material points' velocity; they are the position changes of the different material points in special structures or defects in the current configuration.

\section{Basic Laws of Shale under Hydration}

3.1. Hydration Stress of Shale. For shale, each force increment per unit volume $\Delta F$ produces the volume deformation energy. According to the energy equivalent principle, the total deformation per unit volume equals the volume deformation energy under the condition of certain stress, which is hydration stress $\sigma_{h}$.

All clay minerals (per unit volume $\delta^{\prime}$ ) do the work $W_{t}^{i}$ in the direction $i$, because of hydration expansion.

$$
\begin{aligned}
W_{t}^{i}= & W^{i}+W_{c S}^{i}=\varepsilon_{h_{i}} \sum_{j}\left[h_{j} \Delta F_{j}\left(h_{j}\right) n_{j} \int_{0}^{\pi} P_{j a_{i}} \cos ^{2} a_{i} d a_{i}\right] \\
& +\varepsilon_{h_{i}} h_{c S} \Delta F_{c S}\left(h_{c S}\right) n_{c S} \int_{0}^{\pi} P_{s a_{i}} \cos ^{2} a_{i} d a_{i} .
\end{aligned}
$$

According to the energy equivalent principle, all the work, made by all clay minerals (per unit volume $\delta^{\prime}$ ), equals deformation energy, which is made from the strain in the direction $i$ of shale produced by the component $\sigma_{h_{i i}}$ of hydration expansion $\sigma_{h}$ in the direction $i$.

$$
\begin{aligned}
\frac{1}{2} \sigma_{h_{i i}} \varepsilon_{h_{i}}= & \varepsilon_{h_{i}} \sum_{j}\left[h_{j} \Delta F_{j}\left(h_{j}\right) n_{j} \int_{0}^{\pi} P_{j a_{i}} \cos ^{2} a_{i} d a_{i}\right] \\
& +\varepsilon_{h_{i}} h_{c S} \Delta F_{c S}\left(h_{c S}\right) n_{c S} \int_{0}^{\pi} P_{s a_{i}} \cos ^{2} a_{i} d a_{i} .
\end{aligned}
$$

The deformation density $W^{t}$ produced by hydration stress (per unit volume $\delta^{\prime}$ ) is

$$
\begin{aligned}
W^{t}= & \varepsilon_{h_{i}} \sum_{j}\left[h_{j} \Delta F_{j}\left(h_{j}\right) n_{j} \int_{0}^{\pi} P_{j a_{i}} \cos ^{2} a_{i} d a_{i}\right] \\
& +\varepsilon_{h_{i}} h_{c S} \Delta F_{c S}\left(h_{c S}\right) n_{c S} \int_{0}^{\pi} P_{s a_{i}} \cos ^{2} a_{i} d a_{i},
\end{aligned}
$$

whose material form in reference configuration is 


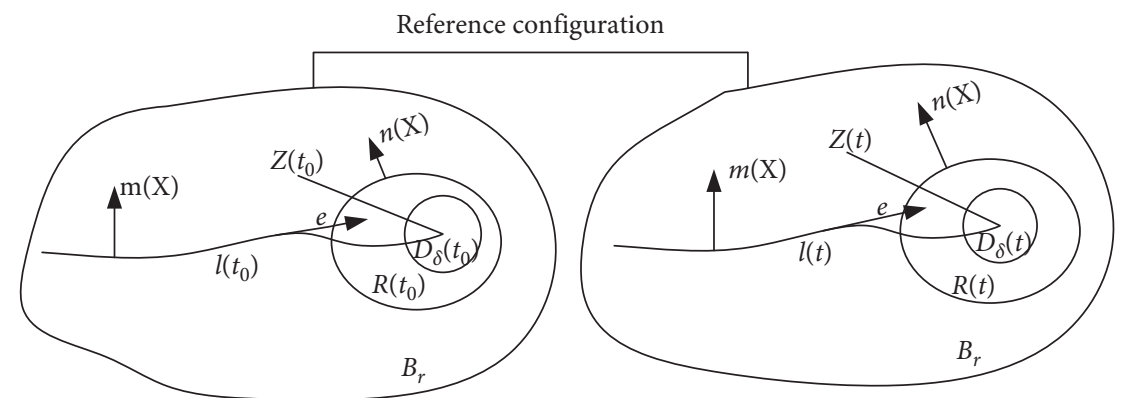

FIGURE 2: Control volume evolution and crack propagation in reference configuration.

$$
\begin{aligned}
W^{w}= & J_{F} F^{-1} \varepsilon_{h_{i}} \sum_{j}\left[h_{j} \Delta F_{j}\left(h_{j}\right) n_{j} \int_{0}^{\pi} P_{j a_{i}} \cos ^{2} a_{i} d a_{i}\right] \\
& +J_{F} F^{-1} \varepsilon_{h_{i}} h_{c S} \Delta F_{c S}\left(h_{c S}\right) n_{c S} \int_{0}^{\pi} P_{s a_{i}} \cos ^{2} a_{i} d a_{i}
\end{aligned}
$$

where $J_{F}$ is the deformation gradient value of the Jacobian determinant.

According to (12), the stress tensor of shale hydration is

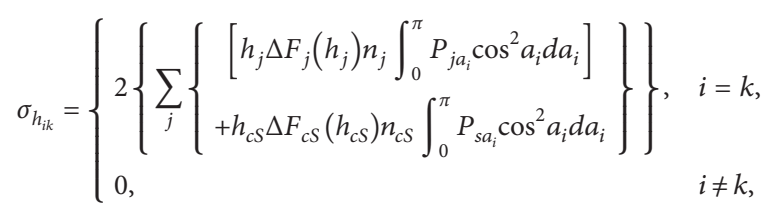

whose material form in the reference configuration is

$$
\mathbf{T}^{h_{i k}}=J_{F} \sigma_{h_{i k}} \mathbf{F}^{-T},
$$

where $T^{h_{i k}}$ is the stress tensor of shale hydration in reference configuration.

3.2. Equilibrium Equation of Deformation Force. The deformation force balance equation (9) is

$$
\begin{aligned}
\nabla \cdot \mathbf{T}+\mathbf{b} & =0, \\
\mathbf{T F}^{T} & =\mathbf{F}(\mathbf{T})^{T},
\end{aligned}
$$

where $\mathbf{b}=\rho_{0} \ddot{y}+\mathbf{f}, \rho_{0}$ is the density, and $\mathbf{f}$ is the volume force.

In (17), the deformation force $T$ consists of two parts, because the material particles in shale are not only affected by overlying rock loadings, but also affected by the hydration stress $[43,44]$. Accordingly, we have the following form:

$$
\mathbf{T}=\mathbf{T}^{J}+\mathbf{T}^{h_{i k}}
$$

where $\mathbf{T}^{J}$ is the first Piola-Kirchhoff stress.

\section{Basic Law of Configuration Force in Shale under Hydration}

4.1. Total Work of Migrating Control Volume. Because the current control body $R(t)$ is subjected to hydration and overlying rock loadings, the total work on it can be expressed as

$$
\begin{aligned}
W(R)= & \int_{R} \dot{e} \mathrm{~d} V=\int \mathbf{T}^{J} \mathbf{n} \cdot \overline{\mathbf{u}} \mathrm{d} S+\int_{R(t)} \mathbf{b} \cdot \bar{u} \mathrm{~d} V \\
& +\int_{\partial R(t)} \mathbf{T}^{h_{i k}} \mathbf{n} \cdot \overline{\mathbf{u}} \mathrm{d} S \\
& +\int_{R(t)} \mathbf{T}^{h_{i k}} \cdot \overline{\mathbf{u}} \mathrm{d} V+\int_{\partial R(t)} \mathbf{C n} \cdot \mathbf{u} \mathrm{d} S+\int_{R(t)} \mathbf{g} \cdot \mathbf{u} \mathrm{d} V .
\end{aligned}
$$

For the migrating control volume $R(t)$, the work of the deformation force and the configuration force should be considered simultaneously. Material accretion has no connection with the body deformation; thus, the configuration stress $\mathbf{C}$ and the configuration body force $\mathbf{g}$ are accompanied by the migrating control volume. It is reasonable that they work over the velocity $u$ in the reference configuration, because the intrinsic material description of the deformed body $y(R(t))$ is nonexistent owing to the dependence of $R(t)$ on $t$. Furthermore, material points are constantly being removed and added through the boundary of the control body [36] $\partial R(t)$. On this basis, it is rational that the deformation tractive force $\mathbf{T}^{J}$ and the deformation force $\mathbf{b}$ perform work over velocity $\bar{u}$, which takes the coupled motion of material points and boundary evolution of $\partial R(t)$ into account, and the hydration stress $\mathbf{T}^{h_{i k}}$ performs work over $\bar{u}$ at the boundary evolution of $\partial R(t)$ and performs work over $\dot{y}$ in the interior of migrating control volume.

In the interior of migrating control volume $u=0$, (19) can be simplified as

$$
\begin{aligned}
W(R)= & \int_{\partial R(t)} \mathbf{T}^{J} \mathbf{n} \cdot \overline{\mathbf{u}} \mathrm{d} S+\int_{R(t)} \mathbf{b} \cdot \dot{\mathbf{y}} \mathrm{d} V+\int_{\partial R(t)} \mathbf{T}^{h_{i k}} \mathbf{n} \cdot \overline{\mathbf{u}} \mathrm{d} S \\
& +\int_{R(t)} \mathbf{T}^{h_{i k}} \cdot \overline{\mathbf{u}} \mathrm{d} V+\int_{\partial R(t)} \mathbf{C n} \cdot \mathbf{u} \mathrm{d} S .
\end{aligned}
$$

4.2. Derivation of Configuration Force Equilibrium Equation. In this subsection, the configuration force balance equation is based on the rotation transformation of the arbitrary rigid body in the spatial observer. Based on the basic idea of Gurtin, the configuration force balance and configuration moment balance are obtained under an overlying rock and hydration loadings. The time-dependent change is considered in translation (see Figure 3). 


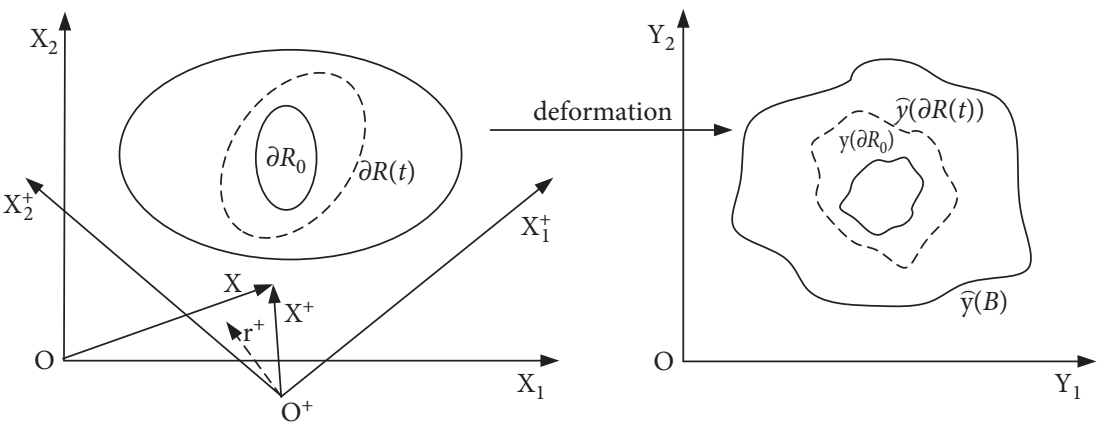

FIgURE 3: Migrating control volume in spatial observers.

$$
\mathbf{X} \longrightarrow \mathbf{X}^{+}=\mathbf{Q}(t) \mathbf{X}+\left(t-t_{0}\right) \mathbf{A},
$$

where $\mathbf{A}$ is a constant and $\mathbf{Q}(t)$ is the orthogonal matrix, which represents transformation between the reference configuration and the current configuration. The equivalent equation (30) can be expressed as

$$
\mathbf{X}=\mathbf{Q}^{T}\left[\mathbf{X}^{+}-\left(t-t_{0}\right) \mathbf{A}\right]=\mathbf{Q}^{T} \mathbf{r}^{+},
$$

where $\mathbf{r}^{+}$denotes the translation metric of vector $\mathbf{X}$ in the new spatial observation, and $\mathbf{Q}^{T}$ is the transpose matrix of $\mathbf{Q}$.

By (21), the velocity of the migrating control volume boundary $\partial R(t)$ in the reference configuration can be written as

$$
\mathbf{u}^{+}=\frac{\partial \widehat{\mathbf{X}}^{+}\left(\xi_{1}, \xi_{2}, t\right)}{\partial t}=\mathbf{A}+\mathbf{Q u}+\dot{\mathbf{Q} X}
$$

By (22), (23) may be rewritten in the form

$$
\mathbf{u}^{+}=\mathbf{A}+\mathbf{Q u}+\dot{\mathbf{Q}} \mathbf{Q}^{T} \mathbf{r}^{+}=\mathbf{A}+\mathbf{Q u}+\mathbf{\Omega} \mathbf{r}^{+}=\mathbf{A}+\mathbf{Q u}+\dot{\theta} \times \mathbf{r}^{+},
$$

where $\Omega=\dot{\mathbf{Q}} \mathbf{Q}^{T}$ is the angular velocity tensor of the new spatial observer moving relative to the old spatial observer angular velocity tensor, $\dot{\theta}=-(1 / 2) \epsilon: \Omega$ is the axis vector corresponding to $\Omega$, and $\epsilon$ is the displacement tensor.

According to the definition of migrating control volume, compared with the old spatial observer $X_{1} O X_{2}$, the boundary of the control body migrates with the velocity $u$, but the internal material points are static. Relative to the new spatial observer $\mathrm{X}_{1}^{+} \mathrm{OX}_{2}^{+}$, material points inside the control volume migrate with the velocity $\mathbf{A}+\dot{\theta} \times \mathbf{r}^{+}$, and the boundary of the control volume migrates with the velocity $\mathbf{A}+\mathbf{Q u}+\dot{\theta} \times \mathbf{r}^{+}$.

By the transformation relation equation (21), we can define the following physical quantities:

$$
\begin{aligned}
& \mathbf{F}^{+}=\frac{\partial \mathbf{y}}{\partial \mathbf{X}^{+}}=\mathbf{F} \mathbf{Q}^{T}, \\
& \dot{\mathbf{y}}^{+}=\left|\frac{\partial \mathbf{y}}{\partial t}\right|_{X^{+}}=\dot{\mathbf{y}}-\mathbf{F Q}^{T}\left(\mathbf{A}+\dot{\boldsymbol{\theta}} \times \mathbf{r}^{+}\right) .
\end{aligned}
$$
$[20]$ is

$$
\overline{\mathbf{u}}_{c}^{+}=\frac{\partial \widehat{\mathbf{y}}\left(\widehat{\mathbf{X}}^{+}\left(\xi_{1}, \xi_{2}, t\right), t\right)}{\partial t}=\dot{\mathbf{y}}^{+}+\mathbf{F}^{+} \mathbf{u}^{+} .
$$

The transformation of $\bar{u}_{c}^{+}$into the reference configuration is obtained

$$
\overline{\mathbf{u}}^{+}=\mathbf{Q} \overline{\mathbf{u}}_{c}^{+}=\mathbf{Q}\left(\dot{\mathbf{y}}^{+}+\mathbf{F}^{+} \mathbf{u}^{+}\right) .
$$

Equations (23), (24), (4), and (27) yield the following form:

$$
\overline{\mathbf{u}}^{+}=\mathbf{Q} \overline{\mathbf{u}}_{c}^{+}=\mathbf{Q}\left(\dot{\mathbf{y}}^{+}+\mathbf{F}^{+} \mathbf{u}^{+}\right)=\mathbf{Q} \overline{\mathbf{u}} .
$$

The transformation of $\mathbf{n}$ and $\dot{y}$ into the configuration is

$$
\begin{aligned}
& \mathbf{n} \longrightarrow \mathbf{n}^{+}=\mathbf{Q n}, \\
& \dot{\mathbf{y}} \longrightarrow \mathbf{Q} \dot{\mathbf{y}} .
\end{aligned}
$$

In the reference configuration, configuration forces and deformational forces are defined as

$$
\begin{aligned}
\mathbf{b}^{+} & =\mathbf{Q} \mathbf{b}, \\
\mathbf{g}^{+} & =\mathbf{Q g}, \\
\mathbf{T}^{+} \mathbf{n}^{+} & =\mathbf{Q}(\mathbf{T} \mathbf{n}), \\
\mathbf{C}^{+} \mathbf{n}^{+} & =\mathbf{Q}(\mathbf{C n}), \\
\mathbf{T}_{i k}^{h^{+}} & =\mathbf{Q} \mathbf{T}^{h_{i k}}, \\
\mathbf{T}^{J_{+}} & =\mathbf{Q} \mathbf{T}^{J} .
\end{aligned}
$$

Because of the relative translation and rotation between the new reference configuration and the current reference configuration, configuration body moment $\mathbf{h}$ is introduced. The work it does can be expressed as $\mathbf{Q h} \cdot \dot{\theta}$ in the reference configuration. Configuration force $g^{+}$does work at the velocity of $\mathbf{A}+\dot{\theta} \times \mathbf{r}^{+}$in the new spatial observer. Deformation body force $\mathbf{b}^{+}$does work at the velocity of $\mathbf{Q} \dot{y}$. Hydration stress $\mathbf{T}^{h_{i k}+}$ does work at the velocity of $\bar{u}$ on the surface of the migrating control volume; it also performs work at the velocity of $\dot{y}$ inside the control volume.

In the reference configuration, the work done on the migrating control volume is 


$$
\begin{aligned}
& W_{m}(R(t))\left[X^{+}\right]=\int_{\partial R(t)}\left(\mathbf{T}^{J+} \mathbf{n}^{+}\right) \cdot \overline{\mathbf{u}}^{+} \mathrm{d} S+\int_{R(t)} \mathbf{b}^{+} \cdot \mathbf{Q} \dot{\mathbf{y}} \mathrm{d} V+\int_{\partial R(t)} \mathbf{C}^{+} \mathbf{n}^{+} \cdot \mathbf{u}^{+} \mathrm{d} S
\end{aligned}
$$

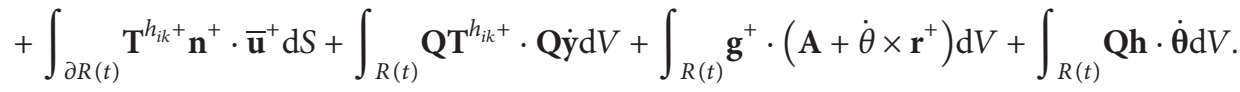

By (24) to (30), (31) may be rewritten as

$$
\begin{aligned}
& W_{m}(R(t))\left[X^{+}\right]=\int_{\partial R(t)} \mathbf{T}^{J} \mathbf{n} \cdot \bar{u} \mathrm{~d} S+\int_{R(t)} \mathbf{b} \cdot \dot{y} \mathrm{~d} V+\int_{\partial R(t)} \mathbf{C n} \cdot \mathbf{u} d S \\
& +\int_{\partial R(t)}(\mathbf{Q C n}) \cdot\left(\mathbf{A}+\dot{\theta} \times \mathbf{r}^{+}\right) \mathrm{d} S+\int_{\partial R(t)} \mathbf{T}^{h_{i k}} \mathbf{n} \cdot \mathbf{u d} S+\int_{\partial R(t)}\left(\mathbf{Q} \mathbf{T}^{h_{i k}} \mathbf{n}\right) \cdot\left(\mathbf{A}+\dot{\theta} \times \mathbf{r}^{+}\right) \mathrm{d} S \\
& +\int_{R(t)} \mathbf{T}^{h_{i k}} \cdot \dot{y} \mathrm{~d} V+\int_{R(t)} \mathbf{Q g} \cdot\left(\mathbf{A}+\dot{\theta} \times \mathbf{r}^{+}\right) \mathrm{d} V+\int_{R(t)} \mathbf{Q h} \cdot \dot{\theta} \mathrm{d} V .
\end{aligned}
$$

As a consequence of the invariance under changes, (20) and (32) yield the following form:

$$
\begin{aligned}
\int_{\partial R(t)} & \left(\mathbf{C}+\mathbf{T}^{h_{i k}}\right) \mathbf{n} \cdot\left(\mathbf{A}+\dot{\theta} \times \mathbf{r}^{+}\right) \mathrm{d} S+\int_{R(t)} \mathbf{g} \cdot\left(\mathbf{A}+\dot{\theta} \times \mathbf{r}^{+}\right) \mathrm{d} V \\
& +\int_{R(t)} \mathbf{h} \cdot \dot{\theta} \mathrm{d} V=0 .
\end{aligned}
$$

According to divergence theorem, (33) can be written as

$$
\begin{aligned}
& \mathbf{A} \cdot \int_{R(t)}\left(\nabla\left(\mathbf{C}+\mathrm{T}^{h_{i k}}\right)+\mathbf{g}\right) \mathrm{d} V \\
& +\dot{\theta} \cdot\left(\begin{array}{c}
\int_{R(t)}\left(\mathbf{h}-\epsilon:\left(\mathbf{C}+\mathbf{T}^{h_{i k}}\right) \times \mathbf{r}^{+}\right) \mathrm{d} V \\
+\int_{R(t)} \mathbf{r}^{+} \times \int_{R(t)}\left(\nabla\left(\mathbf{C}+\mathbf{T}^{h_{i k}}\right)+\mathbf{g}\right) \mathrm{d} V
\end{array}\right)=0 .
\end{aligned}
$$

For any $A$ and $\dot{\theta}$, the above formula is always established, which requires

$$
\begin{array}{r}
\nabla\left(\mathbf{C}+\mathbf{T}^{h_{i k}}\right)+\mathbf{g}=0, \\
\mathbf{h}-\epsilon:\left(\mathbf{C}+\mathbf{T}^{h_{i k}}\right) \times \mathbf{r}^{+}=0 .
\end{array}
$$

Formula (35) is the configuration force equilibrium equation of shale under hydration. Considering the rotating transformation of the spatial observer, the configuration moment equilibrium equation is obtained.

4.3. Equation of Configuration Stress C. By (4), the total work equation (20) on the migrating control volume can be written as

$$
\begin{aligned}
W_{m}(R(t))= & \int_{\partial R(t)} \mathbf{T}^{J} \mathbf{n} \cdot \dot{y} \mathrm{~d} S+\int_{R(t)} \mathbf{b} \cdot \dot{y} \mathrm{~d} V+\int_{\partial R(t)} \mathbf{T}^{h_{i k}} \mathbf{n} \cdot \dot{y} \mathrm{~d} S \\
& +\int_{R(t)} \mathbf{T}^{h_{i k}} \cdot \dot{y} \mathrm{~d} V+\int_{\partial R(t)}\left(\mathbf{C}+\mathbf{F}^{T} \mathbf{T}^{J}+\mathbf{F}^{T} \mathbf{T}^{h_{i k}}\right) \mathbf{n} \cdot \mathbf{u} d S .
\end{aligned}
$$

The last part is the configuration work. The magnitude of configuration work is only related to the amount of material, not to the manner in which it increases or decreases. In other words, it is only related to the normal component $\mathbf{U}=\mathbf{n} \cdot \mathbf{u}$ of the evolutionary velocity of the interface $\mathbf{u}$, but it is independent of its tangential component, for any of the tangential component $t$; that is,

$$
\int_{\partial R(t)}\left(\mathbf{C}+\mathbf{F}^{T} \mathbf{T}^{J}+\mathbf{F}^{T} \mathbf{T}^{h_{i k}}\right) \mathbf{n} \cdot \mathbf{t d} S=0, \text { for all } t,
$$

which equals

$$
\mathbf{C}+\mathbf{F}^{T} \mathbf{T}^{J}+\mathbf{F}^{T} \mathbf{T}^{h_{i k}}=\bar{w} \mathbf{I}
$$

where $\bar{w}$ is an undetermined parameter, which acts on the increase or decrease of material boundaries. By (38), (36) can be rewritten as

$$
\begin{aligned}
W_{m}(R(t))= & \int_{\partial R(t)} \mathbf{T}^{J} \mathbf{n} \cdot \dot{y} \mathrm{~d} S+\int_{R(t)} \mathbf{b} \cdot \dot{y} \mathrm{~d} V+\int_{\partial R(t)} \mathbf{T}^{h_{i k}} \mathbf{n} \cdot \dot{y} \mathrm{~d} S \\
& +\int_{R(t)} \mathbf{T}^{h_{i k}} \cdot \dot{y} \mathrm{~d} V+\int_{\partial R(t)} \bar{w} \mathbf{U} \mathrm{d} S .
\end{aligned}
$$


Following the line of Gurtin, the second law of thermodynamics is still applicable in the framework of configuration forces applied to shale under hydration.

$$
\begin{aligned}
& \frac{\mathrm{d}}{\mathrm{d} t} \int_{R(t)} W^{w} \mathrm{~d} V+K(R(t)) \leq W_{m}(R(t))=\int_{\partial R(t)} \mathbf{T}^{J} \mathbf{n} \cdot \dot{y} \mathrm{~d} S \\
& \quad+\int_{R(t)} \mathbf{b} \cdot \dot{y} \mathrm{~d} V+\int_{\partial R(t)} \mathbf{T}^{h_{i k}} \mathbf{n} \cdot \dot{y} \mathrm{~d} S+\int_{R(t)} \mathbf{T}^{h_{i k}} \cdot \dot{y} \mathrm{~d} V \\
& \quad+\int_{\partial R(t)} \bar{w} \mathbf{U d} S .
\end{aligned}
$$

In (40), $K(R(t))$ is the rate of kinetic energy.

$$
K(R(t))=\frac{\mathrm{d}}{\mathrm{d} t} \int_{R(t)} \mathbf{k} \mathrm{d} V-\int_{\partial R(t)} \mathbf{k} \mathbf{U}_{\partial R(t)} \mathrm{d} S=\int_{R(t)} \dot{\mathbf{k}} \mathrm{d} V .
$$

In (41), $\mathbf{k}=\rho_{0} \dot{\mathbf{y}}^{2} / 2$ is the kinetic energy density. Because $K(R(t))$ denotes the contribution of inertial forces, the right part of (40) does not include their work.

By the transport theorem, therefore,

$$
\frac{\mathrm{d}}{\mathrm{d} t} \int_{R(t)} \mathbf{W}^{w} \mathrm{~d} V=\int_{R(t)} \dot{W}^{w} \mathrm{~d} V+\int_{\partial R(t)} \mathbf{W}^{w} \mathbf{U}_{\partial R(t)} \mathrm{d} S .
$$

By (42), (40) can be rewritten as

$$
\begin{aligned}
& \int_{R(t)} \dot{W}^{w} \mathrm{~d} V+K(R(t)) \leq \int_{\partial R(t)} \mathbf{T}^{J} n \cdot \dot{y} \mathrm{~d} S+\int_{R(t)} \mathbf{b} \cdot \dot{y} \mathrm{~d} V \\
& +\int_{\partial R(t)} \mathbf{T}^{h_{i k}} \mathbf{n} \cdot \dot{y} \mathrm{~d} S+\int_{R(t)} \mathbf{T}^{h_{i k}} \cdot \dot{y} \mathrm{~d} V+\int_{\partial R(t)}\left(\bar{w}-W^{w}\right) \mathbf{U d} S .
\end{aligned}
$$

Equation (43) is applicable to any control body. No matter what the migrating velocity $U_{\partial R(t)}$ is, the above form is constantly set up. This requires

$$
\bar{w}=W^{w} .
$$

Equations (44) and (54) yield

$$
\mathbf{C}=W^{w} \mathbf{I}-\mathbf{F}^{T} \mathbf{T}^{J}-\mathbf{F}^{T} \mathrm{~T}^{h_{i k}} .
$$

So far, we have obtained the configuration stress equation of shale under hydration, also known as the energymomentum tensor, which is the driving force of rock crack propagation in the underground.

\section{Study on Dynamic Fracture of Shale under Hydration by Configuration Force}

5.1. Equilibrium Equation of the Crack Tip. For shale under hydration, the integral form of the equilibrium equation (17) is

$$
\begin{aligned}
\oint_{t i p} \operatorname{Tnd} S+\mathbf{b}_{t i p}^{i}+\mathbf{b}_{t i p}^{e} & =0, \\
\mathbf{b}_{t i p}^{i} & =\lim _{\delta \longrightarrow 0} \int_{D_{\delta}(t)} \mathbf{f} \mathrm{d} V, \\
\mathbf{b}_{t i p}^{e} & =\lim _{\delta \longrightarrow 0} \int_{D_{\delta}(t)}\left(-\rho_{0} \ddot{\mathbf{y}}\right) \mathrm{d} V .
\end{aligned}
$$

Normally, the work done by the volume force $f$ is finite, so $b_{\text {tip }}^{i}=0$; the integral equation can be rewritten as

$$
\oint_{t i p} \operatorname{Tn} \mathrm{d} S+\mathbf{b}_{t i p}^{e}=0
$$

Similarly, the configuration equation of force equilibrium in integral form can be obtained:

$$
\oint_{t i p} \nabla\left(\mathbf{C}+\mathbf{T}^{h_{i k}}\right) \mathbf{n d} S+\mathbf{g}_{t i p}^{i}+\mathbf{g}_{t i p}^{e}=0 .
$$

In (48), $b_{t i p}^{e}$ is the inertial force, which is centralized at the crack tip, $g_{\text {tip }}^{i}$ is the internal part of the configuration force, and $g_{t i p}^{e}$ is the inertial part of the configuration force.

5.2. Deduction of the Energy Release Rate at the Crack Tip. According to Gurtin [31], the second law can be applied to migrating control volumes, and the energy dissipation $\Gamma\left(D_{\delta}(t)\right)$ on the crack tip disc of the control body $D_{\delta}(t)$ can be written as

$$
\Gamma\left(D_{\delta}(t)\right)=W\left(D_{\delta}(t)\right)-\frac{\mathrm{d}}{\mathrm{d} t} \int_{D_{\delta}(t)} W^{w} \mathrm{~d} V .
$$

In (49), the first part on the right is the total work, and the second part on the right is the total potential energy change. As the crack tip is included in $D_{\delta}(t)$, the total work $W\left(D_{\delta}(t)\right)$ can be written as

$$
W\left(D_{\delta}(t)\right)=W_{m}\left(D_{\delta}^{r}(t)\right)+W\left(\ell_{\delta}(t)\right)+W_{t i p}
$$

In (50), $W_{m}\left(D_{\delta}^{r}(t)\right)$ is the work on the regular region $D_{\delta}^{r}(t)$ in $D_{\delta}(t)$ (except the region of the crack tip points), $W_{t i p}$ is the work at the infinitesimal singular crack tip, and $W\left(\ell_{\delta}(t)\right)$ is the work on the crack surface. Here, $W_{m}\left(D_{\delta}^{r}(t)\right)$ can be expressed as

$$
\begin{aligned}
W_{m}\left(D_{\delta}^{r}(t)\right)= & \int_{\partial D_{\delta}^{r}(t)} \mathbf{T}^{J} \mathbf{n} \cdot \bar{u} \mathrm{~d} S+\int_{D_{\delta}^{r}(t)} \mathbf{b} \cdot \dot{y} \mathrm{~d} V \\
& +\int_{\partial D_{\delta}^{r}(t)} \mathbf{T}^{h_{i k}} \mathbf{n} \cdot \bar{u} \mathrm{~d} S \\
& +\int_{D_{\delta}^{r}(t)} \mathbf{T}^{h_{i k}} \cdot \dot{y} \mathrm{~d} V+\int_{\partial D_{\delta}^{r}(t)} \mathbf{C n} \cdot \mathbf{u d} S .
\end{aligned}
$$

The work $W\left(\ell_{\delta}(t)\right)$ is 


$$
\begin{aligned}
W\left(\ell_{\delta}(t)\right)= & -\int_{\ell_{\delta}(t)} \llbracket \bar{u} \mathbf{T}^{J} \rrbracket \cdot \mathbf{m} \mathrm{d} S-\int_{\ell_{\delta}(t)} \llbracket \mathbf{u C} \rrbracket \cdot \mathbf{m} \mathrm{d} S \\
& -\int_{\ell_{\delta}(t)} \llbracket \bar{u} \mathbf{T}^{h_{i k}} \rrbracket \cdot \mathbf{m} \mathrm{d} S .
\end{aligned}
$$

Noting that the migrating velocity $\mathbf{u}$ on the crack surface $\ell_{\delta}(t)$ is zero, that is, although the control volume migrates, particles pass through the crack surface, the above form can be reduced to

$$
\begin{aligned}
W\left(\ell_{\delta}(t)\right) & =-\int_{\ell_{\delta}(t)} \llbracket \mathbf{T}^{\mathrm{J}} \dot{y} \rrbracket \cdot \mathbf{m} \mathrm{d} S-\int_{\ell_{\delta}(t)} \llbracket \mathbf{T}^{h_{i k}} \dot{y} \rrbracket \cdot \mathbf{m} \mathrm{d} S \\
W_{t i p} & =\mathbf{b}_{t i p}^{e} \bar{v}+\mathbf{g}_{t i p}^{e} \nu .
\end{aligned}
$$

On the right side of (54), the first part is work done by the inertial configuration force at the crack tip, due to the crack propagation. The crack tip has no fixed material points, so the conjugate velocity of the inertial force is the deformation velocity of the crack tip, not the material velocity. The second part is the work done by the inertial configuration force, and its conjugate velocity is the evolutionary velocity of the crack tip.

Equation (49) can be reduced to

$$
\begin{aligned}
\Gamma\left(D_{\delta}(t)\right)= & W_{m}\left(D_{\delta}^{r}(t)\right)+W\left(\ell_{\delta}(t)\right)+W_{t i p} \\
& -\frac{\mathrm{d}}{\mathrm{d} t} \int_{D_{\delta}(t)} W^{w} \mathrm{~d} V .
\end{aligned}
$$

In (55), when $\delta \longrightarrow 0, \Gamma_{\text {tip }}$ can be written as

$$
\Gamma_{t i p}=\lim _{\delta \longrightarrow 0} \Gamma\left(D_{\delta}(t)\right)=\lim _{\delta \longrightarrow 0}\left(\begin{array}{l}
W_{m}\left(D_{\delta}^{r}(t)\right)+W\left(\ell_{\delta}(t)\right) \\
+W_{t i p}-\frac{\mathrm{d}}{\mathrm{d} t} \int_{D_{\delta}(t)} W^{w} \mathrm{~d} V
\end{array}\right) .
$$

The limit form of $W_{m}\left(D_{\delta}^{r}(t)\right)$ may be written as

$$
\begin{aligned}
\lim _{\delta \longrightarrow 0} W_{m}\left(D_{\delta}^{r}(t)\right)= & \oint_{t i p} \mathbf{T}^{J} \mathbf{n} \cdot \bar{u} \mathrm{~d} S+\oint_{t i p} \mathbf{C n} \cdot \mathbf{u} \mathrm{d} S+\oint_{t i p} \mathbf{T}^{h_{i k}} \mathbf{n} \cdot \bar{u} \mathrm{~d} S \\
& +\lim _{\delta \longrightarrow 0} \int_{D_{\delta}^{r}(t)} \mathbf{b} \cdot \dot{y} \mathrm{~d} V+\lim _{\delta \longrightarrow 0} \int_{D_{\delta}^{r}(t)} \mathbf{T}^{h_{i k}} \cdot \dot{y} \mathrm{~d} V .
\end{aligned}
$$

The integral functions $\mathbf{b} \cdot \dot{y}$ and $\mathbf{T}^{h_{i k}} \cdot \dot{y}$ are finite in the integral region $D_{\delta}^{r}(t)$, as the crack tip is out of the control volume. Thus, the last two terms are zero, and at the same time, by (2), (4), (6), and (10), when $\delta \longrightarrow 0$,

$$
\begin{aligned}
& \mathbf{u} \longrightarrow \mathbf{v}, \\
& \bar{u} \longrightarrow \bar{v} .
\end{aligned}
$$

By (57) and (58), (56) can be written as

$$
\begin{aligned}
\lim _{\delta \longrightarrow 0} W_{m}\left(D_{\delta}^{r}(t)\right)= & \bar{v} \cdot \oint_{t i p} \mathbf{T}^{J} n \mathrm{~d} S+\mathbf{v} \cdot \oint_{t i p} \mathbf{C n d} S \\
& +\bar{v} \cdot \oint_{t i p} \mathbf{T}^{h_{i k}} \mathbf{n} \mathrm{d} S .
\end{aligned}
$$

Generally, $W\left(\ell_{\delta}(t)\right)$ is also finite on the crack surface. Thus, no matter what hydration and rock boundary conditions exist on the crack surface of the shale under hydration, the limit equation (53) is zero.

By the transport theorem, we get the following form:

$$
\lim _{\delta \rightarrow 0} \frac{\mathrm{d}}{\mathrm{d} t} \int_{D_{\delta}(t)} W^{w} \mathrm{~d} V=0 .
$$

By (54), (59), and (60), (72) may be written as

$$
\begin{aligned}
\Gamma_{t i p}= & \bar{v} \cdot\left(\oint_{t i p}\left(\mathbf{T}^{J}+\mathbf{T}^{h_{i k}}\right) \mathbf{n} \mathrm{d} S+\mathbf{b}_{t i p}^{e}\right) \\
& +v \cdot\left(\oint_{t i p} \operatorname{Cnd} S+\mathbf{g}_{t i p}^{e}\right) .
\end{aligned}
$$

By (18), (46), and (47), (61) may be rewritten as

$$
\Gamma_{t i p}=-\mathbf{g}_{t i p}^{i} \cdot \mathbf{v} \text {. }
$$

The energy dissipation $J_{t i p}$ is obtained in the form

$$
J_{t i p}=\frac{\Gamma_{t i p}}{|\mathbf{v}|}=-\mathbf{g}_{t i p}^{i} \cdot \mathbf{e} .
$$

Equation (63) shows that the negative part of an internal configuration of the crack tip $\mathbf{g}_{\text {tip }}^{i}$ is the energy dissipation rate $J_{\text {tip }}$, when the crack propagates per unit length. It is indicated that the internal force of shale plays a great role in the damage to irreversible shale under hydration. The configuration work done by $\mathbf{g}_{\text {tip }}^{i}$ equals the energy dissipated by the defect migration. The energy release rate of shale under hydration is proved to depend on the constitutive response and hydration stress of shale.

5.3. Deduction of the Configuration Body Force. The theorem of kinetic energy is that the rate of kinetic energy is equal to the power of inertia force. It is noted that the inertial force includes the inertial part of the deformation force and the configuration force; its formula is

$$
\begin{aligned}
\lim _{\delta \longrightarrow 0}( & \left.\frac{\mathrm{d}}{\mathrm{d} t} \int_{D_{\delta}(t)} k \mathrm{~d} V-\int_{\partial D_{\delta}(t)} k(\mathbf{v} \cdot \mathbf{n}) \mathrm{d} S\right) \\
& =-\left(\mathbf{b}_{t i p}^{e} \cdot \bar{v}+\mathbf{g}_{t i p}^{e} \cdot \mathbf{v}\right),
\end{aligned}
$$


where $k$ is the kinetic energy density. According to the transport theorem, the first item on the left of the above form is zero, so (64) can be simplified as

$$
\lim _{\delta \longrightarrow 0} \int_{\partial D_{\delta}(t)} k(\mathbf{v} \cdot \mathbf{n}) \mathrm{d} S=\mathbf{b}_{t i p}^{e} \cdot \bar{v}+\mathbf{g}_{t i p}^{e} \cdot \mathbf{v} .
$$

The following are further transformations of inertial configuration force $\mathbf{b}_{t i p}^{e}$. For the migrating control volume $D_{\delta}(t)$, the equilibrium equation can be written as

$$
\int_{\partial D_{\delta}(t)} \operatorname{Tn} \mathrm{d} S+\int_{D_{\delta}(t)} \mathbf{f} \mathrm{d} V-\int_{D_{\delta}(t)} \dot{\mathbf{p}} \mathrm{d} V=0,
$$

with $\mathbf{p}=\rho_{0} \dot{y}$ denoting the density of linear momentum.

$$
\frac{\mathrm{d}}{\mathrm{d} t} \int_{R(t)} \boldsymbol{\Phi} \mathrm{d} V=\int_{R(t)} \dot{\boldsymbol{\Phi}} \mathrm{d} V+\int_{\partial R(t)} \boldsymbol{\Phi} \mathbf{U} \mathrm{d} S .
$$

By the transport theorem equation (67), (66) can be simplified as

$$
\int_{\partial D_{\delta}(t)} \operatorname{Tnd} S+\int_{D_{\delta}(t)} \mathbf{f d} V-\frac{\mathrm{d}}{\mathrm{d} t} \int_{D_{\delta}(t)} \mathbf{p d} V+\int_{\partial D_{\delta}(t)} \mathbf{p} \mathbf{U d} S=0 .
$$

It is noted that the body force $\mathbf{f}$ is finite; by (13), when $\delta \longrightarrow 0$, the second and third items in (68) are zero, so the limit form can be obtained.

$$
\oint_{\text {tip }} \operatorname{Tnd} S+\oint_{\text {tip }} \mathrm{pUd} S=0
$$

By (69) and (47), the concentrated inertial configuration force $b_{\text {tip }}^{e}$ is equivalent to the following form:

$$
\mathbf{b}_{\text {tip }}^{e}=\oint_{\text {tip }} \mathbf{p}(\mathbf{v} \cdot \mathbf{n}) \mathrm{d} S \text {. }
$$

Equation (70) applied to (65) yields the concentrated configuration force $g_{\text {tip }}^{e}$.

$$
\begin{aligned}
\mathbf{g}_{t i p}^{e} \cdot \mathbf{v}= & \oint_{t i p} k(\mathbf{v} \cdot \mathbf{n}) \mathrm{d} S-\overline{\mathbf{v}} \cdot \oint_{t i p} \mathbf{p}(\mathbf{v} \cdot \mathbf{n}) \mathrm{d} S \\
= & \mathbf{v} \cdot \oint_{t i p}(k-\mathbf{p} \cdot \overline{\mathbf{v}}) \mathbf{n} \mathrm{d} S \\
= & \mathbf{v} \cdot \oint_{t i p} k\left(\frac{1}{2} \rho_{0} \dot{\mathbf{y}}^{2}-\rho_{0} \dot{\mathbf{y}} \cdot \overline{\mathbf{v}}\right) \mathbf{n} \mathrm{d} S=\mathbf{v} \cdot \oint_{t i p} k_{r} \mathbf{n} \mathrm{d} S \\
& -\mathbf{v} \cdot \oint_{t i p} \frac{1}{2} \rho_{0}|\overline{\mathbf{v}}|^{2} \mathbf{n} \mathrm{d} S .
\end{aligned}
$$

In (71),

$$
k_{r}=\frac{1}{2} \rho_{0}|\dot{\mathbf{y}}-\overline{\mathbf{v}}|^{2}=\frac{1}{2} \rho_{0}|\mathbf{F v}|^{2},
$$

which is the kinetic energy at the crack tip. As $\bar{v}$ is finite, the second term of (71) equals zero.

$$
\mathbf{g}_{t i p}^{e} \cdot \mathbf{e}=\mathbf{e} \cdot \oint_{t i p} k_{r} \mathbf{n d} S
$$

At last, (72) and (48) yield the concentrated internal configuration force.

$$
-\mathbf{g}_{t i p}^{i} \cdot \mathbf{e}=\mathbf{e} \cdot \oint_{t i p}\left(\mathbf{C}+\mathbf{T}^{h_{i k}}+k_{r} \mathbf{I}\right) \mathbf{n d} S,
$$

or

$$
-\mathbf{g}_{t i p}^{i} \cdot \mathbf{e}=\mathbf{e} \cdot \oint_{t i p}\left(\left(W^{w}+k_{r}\right) \mathbf{I}-\mathbf{F}^{T} \mathbf{T}^{J}-\mathbf{F}^{T} \mathbf{T}^{h_{i k}}+\mathbf{T}^{h_{i k}}\right) \mathbf{n d} S .
$$

The concentrated configuration body force at the crack tip is obtained. It is the driving force of the crack propagation, which agrees with the classical energy release rate of Moran and Shih [45]. It is indicated that the concentrated configuration body force is related to the constitutive response and hydration stress of shale, and the hydration effect intensifies the propagation of rock cracks, which is the key to affecting the stability of wellbore.

\section{Conclusions}

Aiming at the crack propagation problem of shale under the coupling action of stress field, seepage field, and chemical field, the crack propagation mechanism and fracture problem of shale wellbore under the action of shale load, seepage, and hydration reaction are studied using the theory of configuration mechanics and rock mechanics. We use the configuration force theory to solve the problem of rock crack propagation and rock fracture. The factors considered are more comprehensive, which can better reflect the actual situation and provide a theoretical basis for the study of wellbore stability.

(1) Considering the chemical energy produced by hydration and the mechanical energy under the action of overlying rock load or external force load, the equilibrium process of rock crack growth, the configuration force equation, and the equilibrium equation of the configuration moment at the crack tip are established, under the system of configuration force theory. The configuration stress in the control body of the crack tip, namely, the energy-momentum tensor, is obtained, which is the driving force of rock crack propagation in the underground.

(2) The equilibrium equation and energy dissipation of the crack tip are deduced by the second law, the divergence theorem, and the generalized transport theorem applied to the migrating control volume containing defects. The theoretical deduction shows that the projection of the concentrated internal configuration body force at the crack tip in the opposite direction of the crack is equal to the energy dissipation of the crack tip corresponding to the crack propagation per unit length. The energy and direction of crack propagation are found.

(3) Based on the infinitesimal kinetic energy theorem of migrating control volume at the crack tip, the inertial and internal parts of the concentrated configuration 
force at the crack tip are derived. The results show that the internal configuration force plays a leading role in the irreversible fracture process of rock, which indicates that the energy release rate of rock in the hydration process is related to the constitutive response and hydration stress of shale, and the hydration effect intensifies the propagation of rock.

In this paper, the crack propagation and fracture of shale wall rock under multiphysical coupling are studied, and the configuration force model of shale wall rock crack propagation under multiphysical coupling is established. The additional stress field caused by seepage is considered, but the seepage problems of two-phase flow and multiphase flow are not considered. In underground operation, high temperature will affect the crack propagation of borehole wall rock, so it is necessary to carry out these studies in the next step.

\section{Data Availability}

No data were used to support the study..

\section{Conflicts of Interest}

The authors declare no conflicts of interest.

\section{Acknowledgments}

This work was supported by the National Key Research and Development Program of China (2019YFF0217503).

\section{References}

[1] O. A. Helstrup, Z. Chen, and S. S. Rahman, “Time-dependent wellbore instability and ballooning in naturally fractured formations," Journal of Petroleum Science and Engineering, vol. 43, no. 1-2, pp. 113-128, 2004.

[2] R. Gholami, V. Rasouli, B. Aadnoy, and R. Mohammadi, "Application of in situ stress estimation methods in wellbore stability analysis under isotropic and anisotropic conditions," Journal of Geophysics and Engineering, vol. 12, no. 4, 2015.

[3] R. Gholami, B. Aadnoy, and L. Y. Foon, "A methodology for wellbore stability analysis in anisotropic formations: a case study from the Canning Basin, Western Australia," Journal of Natural Gas Science and Engineering, vol. 37, pp. 341-360, 2017.

[4] E. H. K. Alkamil, H. R. Abbood, R. E. Flori, and A. Eckert, "Case study of wellbore stability evaluation for the Mishrif Formation, Iraq," Journal of Petroleum Science and Engineering, vol. 164, pp. 24-28, 2018.

[5] X. C. Shi, X. Yang, Y. F. Meng, and G. Li, "Wellbore stability analysis in chemically active shale formations," Thermal Science, vol. 20, no. 3, pp. 911-917, 2016.

[6] T. Ma and P. Chen, "A wellbore stability analysis model with chemical-mechanical coupling for shale gas reservoirs," Journal of Natural Gas Science and Engineering, vol. 26, pp. 72-98, 2015.

[7] G. F. Zhen, G. L. Jing, W. J. Hu et al., "The research of the oil base drilling fluid hard brittle shale sidewall instability mechanism," Applied Mechanics and Materials, vol. 513-517, pp. 309-313, 2014.
[8] T. M. Al-Bazali, "The consequences of using concentrated salt solutions for mitigating wellbore instability in shales," Journal of Petroleum Science and Engineering, vol. 80, no. 1, pp. 94101, 2011.

[9] Q. G. Zhang, X. Y. Fan, W. Y. Jia, and Y. C. Liang, "A review of the shale wellbore stability mechanism based on mechanical-chemical coupling theories," Petroleum, vol. 1, no. 2, Article ID S2405656115000292, 2015.

[10] V. Dokhani, M. Yu, and B. Bloys, "A wellbore stability model for shale formations: accounting for strength anisotropy and fluid induced instability," Journal of Natural Gas Science and Engineering, vol. 32, pp. 174-184, 2016.

[11] H. Santos and J. Roegiers, Wellbore Stability: A New Conceptual Approach Based on Energy, S PE 49264, 1998.

[12] A. A. Griffith, "The phenomena of rupture and flow in solid," Philosophical Transactions of the Royal Society of London, vol. 221, pp. 582-593, 1921.

[13] G. R. Irwin, "Analysis of stress and strains near the end of a crack extension force," Journal of Applied Mechanics, vol. 24, pp. 361-364, 1957.

[14] J. M. Kemeny and N. G. W. Cook, "Micromechanics of deformation in rocks," Toughening Mechanisms in Quasi-Brittle Materials, vol. 195, pp. 155-188, 1991.

[15] M. Basista and D. Gross, "The sliding crack model of brittle deformation: an internal variable approach," International Journal of Solids and Structures, vol. 35, no. 5-6, pp. 487-509, 1997.

[16] H. P. Xie, Y. Ju, and L. Y. Li, "Criteria for strength and structural failure of rocks based on energy dissipation and energy release principles," Chinese Journal of Rock Mechanics and Engineering, no. 17, pp. 3003-3010, 2005.

[17] X. G. Chen and Q. Y. Zhan, "Research on the energy dissipation and release in the process of rock shear failure," Caikuang yu Anquan Gongcheng Xuebao/Journal of Mining and Safety Engineering, vol. 27, no. 2, pp. 179-184, 2010.

[18] S. W. Zhang, X. F. Xian, J. P. Zhou et al., "Acoustic emission characteristics and the energy distribution of the shale in Brazilian splitting testing," Journal of China Coal Society, vol. 42, no. S2, pp. 346-353, 2017.

[19] Z. Z. Zhang, Energy Evolution Mechanism during Rock Deformation and Failure, China University of Mining and Technology, Xuzhou, China, 2013.

[20] H. Zhou, Z. Li, and Y. S. Yang, "Unified energy yield criterion of rock," Chinese Journal of Rock Mechanics and Engineering, no. 11, pp. 2170-2184, 2013.

[21] C. Y. Zhao, Research on the Law of Hydraulic Fracture Propagation Based on Entropy Theory, Northeast Petroleum University, Heilongjiang, China, 2011.

[22] X. Zhao, Numerical Simulation and Experimental Study on Three Dimensional Crack Initiation and Propagation of Shale Stimulated by Hydro-Fracture, China University of Mining and Technology, Xuzhou, China, 2017.

[23] Z. J. Li, Research on the Law of Fracture Initiation and Propagation Based on Principle of Minimum Consume Energy, Northeast Petroleum University, Heilongjiang, China, 2011.

[24] J. D. Eshelby, "The Force on an elastic singularity," Philosophical Transactions of the Royal Society A: Mathematical, Physical and Engineering Sciences, vol. 244, no. 877, pp. 87112, 1951.

[25] J. D. Eshelby, “The continuum theory of lattice defects," Solid State Physics, vol. 2, no. 12, pp. 79-144, 1956.

[26] J. D. Eshelby, Energy Relations and the Energy-Momentum Tensor in Continuum Mechanics, McGraw-Hill, New York, NY, USA, 1970. 
[27] J. D. Eshelby, "The elastic energy-momentum tensor," Journal of Elasticity, vol. 5, no. 3-4, pp. 321-335, 1975.

[28] Gé and G. A. Maugin, "Material forces: concepts and applications," Applied Mechanics Reviews, vol. 48, no. 5, pp. 213-245, 1995.

[29] K. Özenç, G. Chinaryan, and M. Kaliske, "A configurational force approach to model the branching phenomenon in dynamic brittle fracture," Engineering Fracture Mechanics, vol. 157, pp. 26-42, 2016.

[30] M. E. Gurtin and P. Podio-Guidugli, "Configurational forces and the basic laws for crack propagation," Journal of the Mechanics and Physics of Solids, vol. 44, no. 6, pp. 905-927, 1995.

[31] M. E. Gurtin and P. Podio-Guidugli, "Configurational forces and a constitutive theory for crack propagation that allows for kinking and curving," Journal of the Mechanics and Physics of Solids, vol. 46, no. 8, pp. 1343-1378, 1998.

[32] M. E. Gurtin, "The nature of configurational forces," Archive for Rational Mechanics and Analysis, vol. 131, pp. 67-100, 1995.

[33] C. Dascalu and G. A. Maugin, "The thermoelastic materialmomentum equation," Journal of Elasticity, vol. 39, no. 3, pp. 201-212, 1995.

[34] M. Epstein and G. A. Maugin, "Thermoelastic material forces: definition and geometric aspects," Comptes rendus de l'Académie des Sciences, vol. 320, pp. 63-65, 1995.

[35] V. K. Kalpakides and C. Dascalu, "On the configurational force balance in thermomechanics," Proceedings of the Royal Society of London A, vol. 458, no. 2028, pp. 3023-3039, 2002.

[36] Q. L. He, L. Z. Wu, and H. J. Yu, "Investigation on thermomechanical fracture in the framework of configurational forces," European Journal of Mechanics, vol. 28, no. 6, pp. 1064-1071, 2009.

[37] C. Kuhn and R. Müller, "A discussion of fracture mechanisms in heterogeneous materials by means of configurational forces in a phase field fracture model," Computer Methods in Applied Mechanics and Engineering, vol. 312, pp. 95-116, 2016.

[38] A. Mohammadipour and K. Willam, "On the application of a lattice method to configurational and fracture mechanics," International Journal of Solids and Structures, vol. 106-107, pp. 152-163, 2017.

[39] R. Ballarini and G. Royer-Carfagni, "A Newtonian interpretation of configurational forces on dislocations and cracks," Journal of the Mechanics and Physics of Solids, vol. 95, pp. 602-620, 2016.

[40] D. Floros, F. Larsson, and K. Runesson, "On configurational forces for gradient-enhanced inelasticity," Computational Mechanics, no. 2, pp. 1-24, 2017.

[41] D. Schneider, E. Schoof, O. Tschukin et al., "Small strain multiphase-field model accounting for configurational forces and mechanical jump conditions," Computational Mechanics, no. 1, pp. 1-18, 2017.

[42] G. Hénap and L. Szabó, "On the configurational-force-based r-adaptive mesh refinement in isogeometric analysis," Finite Elements in Analysis and Design, vol. 124, pp. 1-6, 2017.

[43] Z. Qu and P. Wang, Creep Damage Instability Study of Shale, Science Press, Beijing, China, 2016.

[44] R. P. Steiger, "Leung, Quantitative determination of the mechanical properties of shales," SPE Drilling Engineering, vol. 7, no. 3, pp. 181-185, 1992.

[45] B. Moran and C. F. Shih, "Crack tip and associated domain integrals from momentum and energy balance," Engineering Fracture Mechanics, vol. 27, no. 6, pp. 615-642, 1987. 